9th A. Friedmann International Seminar and

3rd Casimir Symposium 2015

International Journal of Modern Physics: Conference Series

Vol. 41 (2016) 1660121 (7 pages)

(C) The Author(s)

DOI: $10.1142 /$ S2010194516601216

\title{
From Taub-NUT to Kaluza-Klein magnetic monopole
}

\author{
Nematollah Riazi \\ Department of Physics, Shahid Beheshti University, G.C., Evin, \\ Tehran, 19839, Iran \\ n_riazi@sbu.ac.ir \\ S. Sedigheh Hashemi \\ Department of Physics, Shahid Beheshti University, G.C., Evin, \\ Tehran, 19839, Iran \\ se_hashemi@sbu.ac.ir
}

Received 2 September 2015

Revised 28 September 2015

Published 18 March 2016

\begin{abstract}
We present a Kaluza-Klein vacuum solution which closely resembles the Taub-NUT magnetic monopole and we investigate its physical properties as viewed from four spacetime dimensions. We show that the Taub-NUT Kaluza-Klein vacuum solution in five dimensions is a static magnetic monopole. We find that the four dimensional matter properties do not obey the equation of state of radiation and there is no event horizon. A comparison with the available magnetic monopole solutions and the issue of vanishing and negative mass are discussed.
\end{abstract}

Keywords: Kaluza-Klein; Taub-NUT; magnetic monopole.

PACS numbers: $11.10 . \mathrm{Gh}$

\section{Introduction}

One of the oldest ideas that unify gravity and electromagnetism is the theory of Kaluza and Klein which extends space-time to five dimensions. The physical motivation for this unification is that the vacuum solutions of the $(4+1)$ Kaluza-Klein field equations reduce to the $(3+1)$ Einstein field equations with effective matter and the curvature in $(4+1)$ space induces matter in $(3+1)$ dimensional space-time. ${ }^{1}$ With this idea, the four dimensional energy-momentum tensor is derived from the geometry of an exact five dimensional vacuum solution, and the properties of matter such as density and pressure as well as electromagnetic properties are determined

This is an Open Access article published by World Scientific Publishing Company. It is distributed under the terms of the Creative Commons Attribution 4.0 (CC-BY) License. Further distribution of this work is permitted, provided the original work is properly cited. 
by such a solution. In other words, the field equations of both electromagnetism and gravity can be obtained from the pure five-dimensional geometry.

Kaluza's idea was that the universe has four spatial dimensions, and the extra dimension is compactified to form a circle so small as to be unobservable. Klein's contribution was to make a reasonable physical basis for the compactification of the fifth dimension. This school of thinking later led to the eleven-dimensional supergravity theories in 1980s and to the "theory of everything" or ten-dimensional superstrings.

In this paper, we present a vacuum solution of Kaluza-Klein theory in fivedimensional spacetime which is closely related to the Taub-NUT metric. The TaubNUT solution has many interesting features; it carries a new type of charge (NUT charge), which has topological origins and can be regarded as "gravitational magnetic charge", so the solution is known in some other contexts as the Kaluza-Klein magnetic monopole. ${ }^{2}$

The plan of this paper is as follows. In section 2, we briefly discuss the formalism of five dimensional Kaluza-Klein theory and the effective four-dimensional EinsteinMaxwell equations. We will then present a Taub-NUT-like Kaluza-Klien solution and investigate its physical properties in four dimensions in section 3 . In the last section we will draw our main conclusions.

\section{Kaluza-Klein Theory}

Kaluza (1921) and Klein (1926), used one extra dimension to unify gravity and electromagnetism in a theory which was basically five-dimensional general relativity. The theoretical elegance of this idea is revealed by studying the vacuum solutions of Kaluza-Klein equations and the matter induced in the four-dimensional spacetime. ${ }^{3}$ Thus, we are chiefly interested in the vacuum five dimensional Einstein equations. For any vacuum solution, the energy-momentum tensor vanishes and thus $\hat{G}_{A B}=0$ or, equivalently $\hat{R}_{A B}=0$, where $\hat{G}_{A B} \equiv \hat{R}_{A B}-\frac{1}{2} \hat{R} \hat{g}_{A B}$ is the Einstein tensor, $\hat{R}_{A B}$ and $\hat{R}=\hat{g}_{A B} \hat{R}^{A B}$ are the five-dimensional Ricci tensor and scalar, respectively. $\hat{g}_{A B}$ is the metric tensor in five dimensions. Here, the indices $A, B, \ldots$ run over $0 \ldots 4$.

Generally, one can identify the $\mu \nu$ part of $\hat{g}^{A B}$ with $g^{\mu \nu}$, which is the contravariant four dimensional metric tensor, $A_{\mu}$ with the electromagnetic potential and $\phi$ as a scalar field. The correspondence between the above components is

$$
\hat{g}^{A B}=\left(\begin{array}{cc}
g^{\mu \nu} & -\kappa A^{\mu} \\
-\kappa A^{\nu} & \kappa^{2} A^{\sigma} A_{\sigma}+\phi^{2}
\end{array}\right),
$$

where $\kappa$ is a coupling constant for the electromagnetic potential $A^{\mu}$ and the indices $\mu, \nu$ run over $0,1,2,3$.

The five dimensional field equations reduce to the four dimensional field equations ${ }^{4}$

$$
G_{\mu \nu}=\frac{\kappa^{2} \phi^{2}}{2} T_{\mu \nu}^{E M}-\frac{1}{\phi}\left[\nabla_{\mu}\left(\partial_{\nu} \phi\right)-g_{\mu \nu} \square \phi\right],
$$


and

$$
\begin{gathered}
\nabla^{\mu} F_{\mu \nu}=-3 \frac{\partial^{\mu} \phi}{\phi} F_{\mu \nu}, \\
\square \phi=\frac{\kappa^{2} \phi^{3}}{4} F_{\mu \nu} F^{\mu \nu},
\end{gathered}
$$

where $G_{\mu \nu}$ is the Einstein tensor, $T_{\mu \nu}^{E M} \equiv \frac{1}{4} g_{\mu \nu} F_{\rho \sigma} F^{\rho \sigma}-F_{\mu}{ }^{\sigma} F_{\nu \sigma}$ is the electromagnetic energy-momentum tensor and the field strength $F_{\mu \nu}=\partial_{\mu} A_{\nu}-\partial_{\nu} A_{\mu}$. Knowing the five dimensional metric, therefore, leads to a complete knowledge of the four dimensional geometry, as well as the electromagnetic and scalar fields.

\section{Taub-NUT Solution and Kaluza-Klein Magnetic Monopole}

The Taub-NUT solution was first discovered by Taub (1951), and subsequently by Newman, Tamburino and Unti (1963) as a generalization of the Schwarzschild spacetime. $^{5}$

The Kaluza-Klein monopole of Gross and Perry is described by the following metric which is a generalization of the self-dual Euclidean Taub-NUT solution ${ }^{6}$

$$
\mathrm{d} s^{2}=-\mathrm{d} t^{2}+V\left(\mathrm{~d} x^{5}+4 m(1-\cos \theta) \mathrm{d} \phi\right)^{2}+\frac{1}{V}\left(\mathrm{~d} r^{2}+r^{2} \mathrm{~d} \theta^{2}+r^{2} \sin ^{2} \theta \mathrm{d} \phi^{2}\right),
$$

where

$$
\frac{1}{V}=1+\frac{4 m}{r}
$$

This solution has a coordinate singularity at $r=0$ which is called NUT singularity. This can be absent if the coordinate $x^{5}$ is periodic with period $16 \pi m=2 \pi R$, where $R$ is the radius of the fifth dimension. Gross and Perry showed that the Kaluza-Klein theory can contain magnetic monopole solitons which would support the unified gauge theories and allow us for searching the physics of unification.

Here, we introduce a metric which is a vacuum five dimensional solution, having some properties in common with the monopole of Gross and Perry, while some other properties being different. The proposed static metric is given by

$$
\mathrm{d} s_{(5)}^{2}=-\mathrm{d} t^{2}+w(r)\left(\mathrm{d} r^{2}+r^{2} \mathrm{~d} \theta^{2}+r^{2} \sin ^{2} \theta \mathrm{d} \phi^{2}\right)+\frac{k}{w(r)}(\mathrm{d} \psi+Q \cos \theta \mathrm{d} \phi)^{2} .
$$

Here, the extra coordinate is represented by $\psi . k, Q$ are constants and $w(r)$ is an arbitrary function of the radial coordinate $r$. The coordinates take on the usual values with range $r \geq 0,0 \leq \theta \leq \pi, 0 \leq \phi \leq 2 \pi$ and $0 \leq \psi \leq 2 \pi$.

The Ricci scalar associated with the five dimensional metric (6) is given by

$$
R=\frac{1}{2} \frac{1}{w(r)^{3} r^{4}}\left[2 r^{4} w(r) w^{\prime \prime}(r)+4 w^{\prime}(r) w(r) r^{3}-w^{\prime}(r)^{2} r^{4}+k Q^{2}\right],
$$

since we are interested in vacuum solution where the Ricci tensor is zero $\left(R_{A B}=0\right)$ it is necessary but not sufficient to have a zero Ricci scalar $R=0$ which can be 
solved for the function $w(r)$ to give

$$
w(r)=k_{1}+\frac{k_{2}}{r}+\frac{k_{3}}{r^{2}},
$$

where $k_{1}, k_{2}$ and $k_{3}$ are constants. By substituting the function $w(r)$ in the Ricci scalar in Eq. (7) and for having a zero Ricci scalar one can obtain the following constraint between the constants

$$
k Q^{2}+4 k_{1} k_{3}-k_{2}^{2}=0
$$

or

$$
k_{3}=\frac{1}{4 k_{1}}\left(k_{2}^{2}-k Q^{2}\right),
$$

thus the Ricci scalar in terms of the above constants reduces to

$$
R=\frac{1}{2} \frac{r^{2}\left(4 k_{1}-1\right)\left(k Q^{2}-k_{2}^{2}\right)}{\left(k Q^{2}-k_{2}^{2}-k_{2} r-k_{1} r^{2}\right)^{3}} .
$$

In order to have a zero Ricci scalar the two following possibilities can be obtained for the constants

$$
R=0 \Longrightarrow\left\{\begin{array}{l}
k_{1}=\frac{1}{4} \Longrightarrow R_{A B} \neq 0 \\
k_{2}= \pm \sqrt{k} Q \Longrightarrow R_{A B}=0, \quad R_{A B C D} \neq 0
\end{array}\right.
$$

as it is seen from Eq. (13) with $k_{1}=\frac{1}{4}$ the Ricci tensor is not zero and does not give a vacuum solution, thus this case is discarded. However, the second choice $k_{2}= \pm \sqrt{k} Q$ gives a Ricci flat solution with a non-zero Riemann tensor in five dimensions which is our interest. Consequently, the general form of the function $w(r)$ would be given by

$$
w(r)=k_{1} \pm \frac{\sqrt{k} Q}{r} .
$$

In what follows, we take the constants $k_{1}=1, Q=1$ and $k=4 m^{2}$. Therefore, the metric in Eq. (6) reduces to (we choose the minus sign for $w(r)$ )

$$
\begin{aligned}
\mathrm{d} s_{(5)}^{2}= & -\mathrm{d} t^{2}+\left(1-\frac{2 m}{r}\right)\left(\mathrm{d} r^{2}+r^{2} \mathrm{~d} \theta^{2}+r^{2} \sin ^{2} \theta \mathrm{d} \phi^{2}\right) \\
& +\left(\frac{4 m^{2}}{1-\frac{2 m}{r}}\right)(\mathrm{d} \psi+Q \cos \theta \mathrm{d} \phi)^{2} .
\end{aligned}
$$

The gauge field, $A_{\mu}$ and the scalar field $\phi$ deduced from the metric in Eq. (14) with the help of Eq. (1) are

$$
A_{\phi}=\frac{\cos \theta}{\kappa}
$$


and

$$
\phi^{2}=\frac{4 m^{2}}{1-\frac{2 m}{r}}
$$

respectively. The only non-vanishing component for the electromagnetic tensor which is related to the gauge field $A_{\mu}$ by the general statement $F_{\mu \nu}=\partial_{\mu} A_{\nu}-\partial_{\nu} A_{\mu}$ is

$$
F_{\theta \phi}=-F_{\phi \theta}=-\frac{\sin \theta}{\kappa}
$$

which corresponds to a radial magnetic field $B_{r}=\frac{1}{\kappa r^{2}}$ with a magnetic monopole charge $Q_{M}=\frac{1}{\kappa}$. As the radial coordinate $r$ goes to infinity $r \rightarrow \infty$, the scalar field equals $\phi_{0}^{2}=4 m^{2}$ so that the second part of Eq. (2) becomes zero, therefore

$$
G_{\mu \nu}=\frac{\kappa^{2} \phi_{0}^{2}}{2} T_{\mu \nu}^{E M}=8 \pi G T_{\mu \nu}, \quad \text { as } \quad r \rightarrow \infty,
$$

where we have put the speed of light $c$ equal to 1 . By comparing this equation with the ordinary Einstein equation, we obtain $\frac{\kappa^{2} \phi_{0}^{2}}{2}=8 \pi G$, thus the constant $\kappa$ equals $\kappa=\frac{2}{m} \sqrt{\pi G}$. Straightforwardly, the total magnetic monopole charge is given by

$$
Q_{M}=\frac{m}{2} \frac{1}{\sqrt{\pi G}}
$$

The total magnetic flux through any spherical surface centered at the origin is calculated as ${ }^{7}$

$$
\Phi_{B}=\int F=\frac{1}{2} \oint F_{\mu \nu} d \Sigma^{\mu \nu}=\frac{\pi}{\kappa}
$$

where $F_{\mu \nu}$ is the electromagnetic field tensor as mentioned earlier and $\mathrm{d} \Sigma^{\mu \nu}$ is interpreted as an element of two-dimensional surface area. It is investigated from Eq. (20) that the flux of the magnetic monopole is constant (i.e. we have a singular magnetic charge).

The four dimensional metric deduced from Eq. (14) with the use of Eq. (1) leads to the following asymptotically flat spacetime:

$$
\mathrm{d} s_{(4)}^{2}=-\mathrm{d} t^{2}+\left(1-\frac{2 m}{r}\right) \mathrm{d} r^{2}+r^{2}\left(1-\frac{2 m}{r}\right)\left(\mathrm{d} \theta^{2}+\sin ^{2} \theta \mathrm{d} \phi^{2}\right) .
$$

For this metric, the Ricci scalar and the Kretschmann invariant $K$ are given by

$$
R=\frac{6 m^{2}}{r^{4}\left(1-\frac{2 m}{r}\right)^{3}}
$$


and

$$
K=R_{\mu \nu \rho \sigma} R^{\mu \nu \rho \sigma}=\frac{m^{2}}{(r-2 m)^{5}}\left[\frac{2}{r-2 m}+\frac{2 r-3 m}{r^{2}}\right],
$$

it is seen that the four dimensional metric Eq. (21) has two curvature singularities at $r=0$ and $r=2 \mathrm{~m}$. Let us calculate the surface area of a $S^{2}$ hypersurface at constant $t$ and $r$ to clarify the nature of the $r=2 m$ singularity

$$
A(r)=\int \sqrt{g^{(2)}} \mathrm{d} x^{2}=\int r^{2}\left(1-\frac{2 m}{r}\right) \sin \theta \mathrm{d} \theta \mathrm{d} \phi=4 \pi r^{2}\left(1-\frac{2 m}{r}\right) .
$$

It can be seen that the surface area $A(r)$ becomes zero at $r=2 m$. This means that $r=2 m$ hypersurface is not an event horizon but is just the origin. For the case $r>2 m$ the signature of the metric is proper $(-,+,+,+)$ but for the range $r<2 m$ the signature of the metric will be improper and non-Lorentzian $(-,-,-,-)$, thus the patch $r<2 m$ is excluded from the spacetime. From now on, this spacetime is only considered in the range $r \geq 2 m$. since the range $0<r<2 m$ is omitted from the spacetime, there is only one curvature singularity at $r=2 m$.

The metric (21) can be transformed into the following form, using the radial coordinate transformation $\tilde{r}^{2}=r^{2}\left(1-\frac{2 m}{r}\right)$;

$$
\mathrm{d} s_{(4)}^{2}=-\mathrm{d} t^{2}+\frac{\tilde{r}^{4}}{\left(m^{2}+\tilde{r}^{2}\right)\left(m+\sqrt{m^{2}+\tilde{r}^{2}}\right)^{2}} \mathrm{~d} \tilde{r}^{2}+\tilde{r}^{2} \mathrm{~d} \Omega^{2} .
$$

While the metric (21) is singular at $r=0$ and $r=2 m$, the transformed metric is singular only at $\tilde{r}=0$ which is a curvature singularity.

The properties of the induced matter associated with the above metric can be gained by the Eq. (3). The components of the energy-momentum tensor can be easily calculated for the metric (21). We find that the effective source is like a fluid with an anisotropic pressure. At sufficiently large $r$, the energy-momentum components will tend to zero. The trace of the energy-momentum tensor does not vanish generally, which shows that the effective matter field around the singularity can not be considered as an ultra-relativistic quantum field in contrast to the Kaluza-Klein solitons described in Ref. 8.

Although it is apparent from the 00 component of the $4 D$ metric (21) that the gravitational mass corresponding to this solutions vanishes, it could also be confirmed by calculating the following integrals:

$$
M_{g}(r) \equiv \int\left(T_{0}^{0}-T_{1}^{1}-T_{2}^{2}-T_{3}^{3}\right) \sqrt{g^{(3)}} \mathrm{d}^{3} x,
$$

and

$$
M_{g}(r)=-\frac{1}{8 \pi} \oint_{S} \nabla^{\mu} K^{\nu} \mathrm{d} S_{\mu \nu}
$$

where $g^{(3)}$ is the determinant of the 3 -metric. 


\section{Conclusion}

Inspired by the Taub-NUT solution, we introduced a Kaluza-Klein vacuum solution in $5 D$, which described a magnetic monopole in $(3+1) \mathrm{D}$ spacetime. The gravitational mass of the solution was shown to vanish. The fluid supporting the four dimensional space-time was shown to differ from that of an ultra-relativistic fluid, in contrast with the work by Wesson and Leon. ${ }^{8}$ The pressure is anisotropic in both works.

\section{Acknowledgments}

The authors would like to thank the anonymous referee for helpful comments. N.R. acknowledges the support of Shahid Beheshti University.

\section{References}

1. J. Ponce de Leon and P. S. Wesson, Journal of Math. Phys. 34, 4080 (1993).

2. T. Ortin, Gravity and String (Cambridge University Press, Cambridge, 2004).

3. P. S. Wesson and J. Ponce de Leon. Journal of Math. Phys. 33, 3883 (1992).

4. G. Lessner, Phys. Rev. D. 25, 3202 (1982).

5. D. Baleanu and S. Codoban, General Relativity and Gravitation 31, 497 (1999).

6. D. J. Gross and M. J. Perry, Nucl. Phys. B 29, 226 (1983).

7. R. D. Sorkin, Phys. Rev. Lett. 51, 87 (1983).

8. Paul S. Wesson and J. Ponce de Leon, Classical and Quantum Gravity 11, 1341 (1994). 